\title{
Structure of highly active BSH enzyme with subordinated post-translational excision
}

\author{
Deepak Chand $^{1}{ }^{1}$, Priyabrata Panigrahi ${ }^{1}$, Sureshkumar Ramasamy ${ }^{1}$, Suresh C. G. ${ }^{1}$ \\ ${ }^{1}$ Biochemical Sciences Division, CSIR-National Chemical Laboratory, Pune, India \\ E-mail: chanddeep46@gmail.com
}

The structures of BSH were elucidated by X-ray diffraction, which are clinically important. BSH is involved in the hydrolysis of bile acid in GI tract therefore results in lowering of the cholesterol level in the blood serum. The wild-type EfBSH crystals belonged to space group P21 with unit-cell parameters $a=66.20, b=131.62, c=86.72 \AA$ and $\beta=94.48$. We have reported highest bile acid hydrolysing BSH and investigated the structural features contributing high catalytic activity, supported by the docking and MD simulation studies. These four loops in EfBSH are comprised of residues 21-25 (loop1), 57-64 (loop2), 125-137 (loop3) and 255-269 (loop4). The loop2 of EfBSH has extended in comparison to other BSHs. This disrupted one of the antiparallel $\beta$-sheet of the core conserved $a \beta \beta a-N$ tn-fold in which half of the anti-parallel $\beta$-strand has thus transformed into a large dynamic loop. Functional mutants were also prepared by the site directed mutagenesis to understand the role of binding pocket residues. The investigation of BSH architecture and functional mutant's studies from this study has given insights about hotspots responsible for the high activity and specificity, will help in enzyme engineering in future. The enzyme is important for the probiotic formulation; also studies on enzyme based formulation for the lowering of the serum cholesterol will be appreciated in healthcare. In addition, the putative pre-peptide excision site for autocatalytic processing of Cholylglycine Hydrolases was examined by attaching different $\mathrm{N}$-terminal pre-peptides, which revealed the subordination of the excision site in EfBSH as a result of an amino acid change next to nucleophile serine. It suggests an evolution-mediated subordination or redundancy of the excision site of $\mathrm{EABSH}$, similar to that observed in many other cholylglycine hydrolase family members, subsequent to disappearance of pre-peptide coding part from the bsh gene.

[1] Chand, D., Avinash, V.S., Yadav, Y., Pundle, A.V., Suresh, C.G. and Ramasamy, S., 2017. Biochimica et Biophysica Acta (BBA)-General Subjects, 1861(1), pp.2981-2991.

[2] Rossocha, M., Schultz-Heienbrok, R., von Moeller, H., Coleman, J.P. and Saenger, W., 2005. Biochemistry, 44(15), pp.5739-5748.

[3] Kumar, R.S., Brannigan, J.A., Prabhune, A.A., Pundle, A.V., Dodson, G.G., Dodson, E.J. and Suresh, C.G., 2006. Journal of Biological Chemistry, 281(43), pp.32516-32525.

[4] Chand, D., Ramasamy, S. and Suresh, C.G., 2016. Process Biochemistry, 51(2), pp.263-269.

Keywords: Bile salt hydrolase, $\mathrm{X}$-ray crystallography, Mutagenesis 